\title{
Neutrophil Band Form to Leukocyte Ratio
}

National Cancer Institute

\section{Source}

National Cancer Institute. Neutrophil Band Form to Leukocyte Ratio. NCI Thesaurus. Code C64831.

The determination of the ratio of band neutrophils compared to leukocytes in a blood sample. The measurement may be expressed as a ratio or percentage. 